\title{
Extended Hierarchical Censored Production Rules (EHCPRs) System: An Approach Toward Generalized Knowledge Representation
}

\author{
N.K. Jain \\ Department of Electronics, Zakir Hussain College \\ University of New Delhi, Delhi, INDIA \\ and \\ K.K. Bharadwaj ${ }^{\dagger}$ \\ CAP/LAC/INPE, National Institute for Space Research \\ S.J. Campos, SP, BRAZIL \\ and \\ Norian Marranghello \\ DCCE/IBILCE/UNESP, RIO PRETO, BRAZIL \\ fax: +55-17-224-8692 \\ e-mail:norian@nimitz.dcce.ibilce.unesp.br
}

\begin{abstract}
One common problem in all basic techniques of knowledge representation is the handling of the trade-off between precision of inferences and resource constraints, such as time and memory. Michalski and Winston (1986) suggested the Censored Production Rule (CPR) as an underlying representation and computational mechanism to enable logic based systems to exhibit variable precision in which certainty varies while specificity stays constant. As an extension of CPR, the Hierarchical Censored Production Rules (HCPRs) system of knowledge representation, proposed by Bharadwaj \& Jain (1992), exhibits both variable certainty as well as variable specificity
\end{abstract}

\footnotetext{
${ }^{\dagger}$ Address for correspondence: School of Computer and Systems Sciences, Jawaharlal Nehru University (JNU), New Delhi-110067, INDIA. fax: +55-12-345-6375; e-mail: drkkb@jnuniv.ernet.in. The research of this author is supported by the Brazilian foundation CNPq under Grant No. 301597/95-2 .
} 
and offers mechanisms for handling the trade-off between the two. An HCPR has the form:

Decision If(preconditions)

$$
\begin{aligned}
& \text { Unless(censor) } \\
& \text { Generality(general_information) } \\
& \text { Specificity(specific_information). }
\end{aligned}
$$

As an attempt towards evolving a generalized knowledge representation, an Extended Hierarchical Censored Production Rules (EHCPRs) system is suggested in this paper. With the inclusion of new operators, an Extended Hierarchical Censored Production Rule (EHCPR) takes the general form:

Concept If (Preconditions)

$$
\begin{aligned}
& \text { Unless (Exceptions) } \\
& \text { Generality (General-Concept) } \\
& \text { Specificity (Specific Concepts) } \\
& \text { Has_part (default: structural-parts) } \\
& \text { Has_property (default:characteristic-properties) } \\
& \text { Has_instance (instances). }
\end{aligned}
$$

How semantic networks and frames are represented in terms of an EHCPRs is shown. Multiple inheritance, inheritance with and without cancellation, recognition with partial match, and a few default logic problems are shown to be tackled efficiently in the proposed system.

\section{KEY WORDS}

default logic, frames, hierarchical censored production rule (HCPR), knowledge representation, semantic networks, variable precision logic

\section{INTRODUCTION}

One area in which AI has met success to a certain extent is that of rulebased systems (Buchanan \& Shortliffe, 1984). There are several advantages 
relegated to rule based systems. They are easy to modify and provide modularity in both task and control knowledge (Waterman, 1970; Waterman \& Hayes-Roth, 1978). Newell and Simon (1972) consider production systems to be good candidates for modeling human cognitive processes. Neches et al., (1987) point out further advantages and argue that such systems provide a suitable framework for modeling learning processes. Further, the logic-based approach was shown to have higher expressiveness and also provides an implementation-independent semantics for the knowledge base (Guha \& Lenat, 1994).

The major shortcomings of an ordinary logic based reasoning system is that you cannot tell much about the way you want it to perform its task. For example, you can not give the following instructions:

- Give me a reasonable answer immediately even if it is somewhat general and if there is enough time then give me a more specific answer.

- Give me a reasonable answer immediately. If there is enough time then tell me you are more confident in the answer or change your mind and give me another better answer.

- Give me only a highly certain answer even if it is somewhat general and if there is enough time then give me a more specific answer.

- Give me a reasonable answer immediately even if it is somewhat less certain and if you have enough time then give me a more specific answer.

A system having represented real-world knowledge should also be capable of handling these types of requirements for natural and efficient reasoning. In the real world, both humans and computers often have to reason using insufficient, incomplete, or tentative premises. Moreover, both are subject to constraints of time and memory. Variable precision logic (VPL) (Michalski \& Winston, 1986) is concerned with problems of reasoning with incomplete information, subject to resource constraints and the problem of reasoning efficiently with exceptions. VPL offers mechanisms for handling trade-off between the precision of inferences and computational efficiency of deriving them. Specificity and certainty are the two aspects of precision. Certainty refers to the degree of belief in a statement, whereas specificity refers to the degree of detail of a description. Following Michalski and Winston (1986), a system that gives more specific answers given more time (or resources in 
general) is called a "variable specificity system". A system that gives more certain answers given more time is called a "variable certainty system". There can be various combinations of the two systems, reflecting that specificity and certainty are inversely related; we can gain specificity at the expense of certainty, or vice-versa. Consider a query "What is John doing?" given that it is Sunday. The quickest possible answer to this query may be that "Probably John is working in the yard". A more certain answer taking into account the nice weather is that "Certainly John is working in the yard rather than reading'. Similarly, considering the time of the year, a more specific answer may be that "John is raking leaves'.

Michalski and Winston (1986) have suggested the Censored Production Rule (CPR) as an underlying representational and computational mechanism to enable logic based systems to exhibit variable precision in which certainty varies while specificity stays constant. A CPR has the form, If A Then B Unless $C$, where $C$ (Censor) is the exception condition. Such rules are employed in situations in which the conditional statement 'If A Then B' holds frequently, and the assertion $\mathrm{C}$ holds rarely. By using a rule of this type, we are free to ignore the Censor (exception) condition, when the resources needed to establish its presence are tight, or simply no information is available as to whether it holds or does not hold. As time permits, the Censor condition $\mathrm{C}$ is evaluated establishing the conclusion $\mathrm{B}$ with higher certainty if $\mathrm{C}$ does not hold, or simply changing the polarity of $\mathrm{B}$ to $\sim \mathrm{B}$ if $\mathrm{C}$ holds.

As an example of a censored production rule, If Sunday Then John works in the yard Unless weather is bad, has the interpretation that if it is Sunday and the weather is good, John will work in the yard; and if it is Sunday and the weather is bad, John will not work in the yard.

A CPR may have more than one exception denoting censor. Consider for example the assertion that birds fly (Michalski and Winston, 1986) : $\forall \mathrm{x}$ is $-\operatorname{bird}(\mathrm{x}) \Rightarrow$ flies $(\mathrm{x})$.

This general assertion enables us to express that any newly observed bird flies. But not all birds fly. For example, penguins, ostriches, emus, kiwis, and domestic turkeys do not fly. To include the information we write ( $L$ denotes UNLESS operator): 


$$
\begin{aligned}
\forall x \text { is-bird }(x) & \Rightarrow \\
\operatorname{flies}(x) L & \text { is-penguin (x) } \\
& \vee \text { is-ostrich (x) } \\
& \vee \text { is-emu (x) } \\
& \vee \text { is-kiwi (x) } \\
& \vee \text { is-domestic-turkey (x) }
\end{aligned}
$$

Thus the exceptions are disjunctively linked together as one censor condition. Suppose we generalize these exceptions into one statement for special birds. Then we can write $\forall \mathrm{x}$ is $-\operatorname{bird}(\mathrm{x}) \Rightarrow \operatorname{flies}(\mathrm{x})\lfloor$ is-special-bird (x).

But then the rule is still not entirely correct. Even a flying bird cannot fly when it is dead or sick or has broken wings. Let us characterize all these situations as a bird being in an unusual condition. Then we can write :

$\forall \mathrm{x}$ is - bird( $\mathrm{x}) \Rightarrow$ flies $(\mathrm{x})\lfloor$ (is-special-bird ( $\mathrm{x}) \vee$ is-in-unusual-condition $(\mathrm{x})$ ) where

$$
\begin{aligned}
& \forall x \text { (is-special-bird }(\mathrm{x}) \Leftarrow \\
& \text { is-penguin }(\mathrm{x}) \\
& \vee \text { is-ostrich }(\mathrm{x}) \\
& \vee \text { is-emu (x) } \\
& \vee \text { is-kiwi (x) } \\
& \vee \text { is-domestic-turkey (x) }
\end{aligned}
$$

and

$\forall \mathrm{x}$ is-in-unusual-condition $(\mathrm{x}) \Leftarrow$

$$
\begin{aligned}
& \vee \text { is-dead }(x) \\
& \vee \text { is-sick }(x) \\
& \vee \text { has-broken-wings }(x)
\end{aligned}
$$

Now a bird also cannot fly when its legs are stuck in concrete. This case may also be classified as bird in an unusual condition. Thus, to update our knowledge, we need not change our basic rule; we need only extend our definition of unusual condition: 
$\forall \mathrm{x}$ is-in-unusual-condition $(\mathrm{x}) \Leftarrow$

$\checkmark$ is-dead ( $x$ )

$\checkmark$ is-sick $(x)$

$\checkmark$ has-broken-wings (x).

$\vee$ has-legs-stuck-in-concrete $(x)$.

Thus CPRs facilitate small rule repairs.

Under the Dempster-Shafer Interpretation of VPL, four belief values are associated with each Censored Production Rule (CPR) (Bharadwaj et al., 1994; Haddawy, 1987):

$\mathrm{P} \rightarrow \mathrm{D}\lfloor\mathrm{C}: \alpha, \beta, \gamma, \delta$

such that

(I) $\mathrm{P} \& \sim \mathrm{C} \rightarrow \mathrm{D}: \alpha \quad$ i.e $\operatorname{prob}(\mathrm{D} \mid \mathrm{P} \& \sim \mathrm{C}) \in[\alpha . .1]$

(II) $P \& C \rightarrow \sim D: \beta \quad$ i.e $\operatorname{prob}(\sim D \mid P \& C) \in[\beta$.. 1]

(III) $P \rightarrow D: \gamma \quad$ i.e. $\operatorname{prob}(\mathrm{D} \mid \mathrm{P}) \in[\gamma . .1]$

(IV) $\mathrm{P} \rightarrow \sim \mathrm{D}: \delta \quad$ i.e. $\operatorname{prob}(\sim \mathrm{D} \mid \mathrm{P}) \in[\delta . .1]$, where $\gamma+\delta<=1$.

For example, the following rule might be used to express the fact that I read the paper before going to work unless I oversleep, which occurs once or twice a week :

Weekday-morning $\rightarrow$ Read-paper $\lfloor$ Oversleep : 0.9, 1, 0.6, 0.2,

where the belief factors are interpreted as follows:

- the "0.9" states that on weekday morning when I do not oversleep I read the paper at least 0.9 of the time because there are other factors which would keep me from reading paper; such as the paper boy throwing it on the roof, which are not being considered;

- the " $l$ " states that on weekday mornings when I oversleep I certainly do not read the paper; and

- the " 0.6 " states that I read the paper at least three out of five weekday mornings (because I oversleep at most twice a week).

- the " 0.2 " states that I do not read the paper at least one out of five weekday mornings (because I over sleep at least once a week). 
CPRs-based systems exhibit variable precision in which certainty varies while specificity stays constant and hence, is not able to impart control over the specificity part of a precision in decision making. To address various problems and shortcomings with CPRs system, Bharadwaj and Jain (1992) have introduced a concept of Hierarchical Censored Production Rule (HCPR). An HCPR is a CPR augmented with specificity and generality information and has the following form:

\section{Decision (If precondition)}

(Unless censor_conditions)

(Generality general_information)

(Specificity specific_information)

HCPRs based systems can be made to exhibit variable precision in the reasoning, such that both certainty of belief in a conclusion and its specificity may be controlled by the reasoning process. Such a system has numerous applications in situations in which decisions must be taken in real time and with uncertain information.

Different knowledge base systems can be classified in the following terms of the representation scheme they employ.

i. Systems that employ one of the four formalisms: logic, semantic networks, frames. or production rules. Each having its own merits and demerits and hence none is a universal representation scheme.

ii. Systems that use any one of the four formalisms with a slight change in its syntax and semantics. For example, modal logic (Smyorski, 1985) as a variant of logic, censor production rule for production rule, conceptual graph for semantic network, and schema for frames.

iii. Systems that make use of suitable combinations of two or more of the above four formalisms (Bittencourt, 1990; Brachman \& Levesque, 1985). For example, combination of rule based representation and frame representation has been discussed by Harmon (1990), Sawamoto (1998), and Thraisingham (1989). Krypton (Brachman et al., 1983; 1985) is an example of a system that combines frames with logic, whereas in MANTRA (Calmet et al., 1991) all four formalisms are combined.

iv. In the last category we can place systems that employ a general representation having merits of all four formalisms but demerits of none. 
Hayes (1977) followed a hybrid approach to develop a higher level structure along the lines of a frame and interlinked with a more conventional semantic network. This approach one of the earlier attempts to merge the frame representation and semantic networks. The motive behind the hybrid systems is to combine the merits of two or more independent representations, but at the cost of the uniformity of representation that an individual system may possess. A uniform code of representation supports common procedures and hence, a simplified but enriched inference engine.

The notion of 'common representation' employed by Charniak (1981) associates predicate calculus statements with a frame both for the domain of problem solving and for language comprehension.

An Extended Hierarchical Censored Production Rules (EHCPRs) system is presented in this paper as an attempt toward generalized knowledge representation. The efforts are toward combining the merits of all four formalisms (category [iv] above) and evolve a general representation scheme irrespective of the domain of its use.

\section{HIERARCHICAL CENSORED PRODUCTION RULE (HCPR) SYSTEM}

Following Bharadwaj and Jain (1992), the general form of an HCPR is

Decision (If precondition)

(Unless censor_conditions)

(Generality general_information) (2.1)

(Specificity specific_information)

An HCPRs-tree is a collective and systematical representation of all the related HCPRs about a given problem domain. Here the term "collective" means the set of all rules or definitions related to a most generalized concept in the knowledge base. A HCPR in the HCPRs-tree is the smallest and simplest chunk of knowledge that may be created, modified, or removed without directly affecting the other HCPRs in the HCPRs-tree (because of its declarative nature of representation). A few related HCPRs how they are linked in a HCPR-tree structure, depicted in Fig.l, are given below. This Irepresents a rule-base to find answers to queries of the type "What is $\mathrm{X}$ doing?" when supplied with relevant input data. 


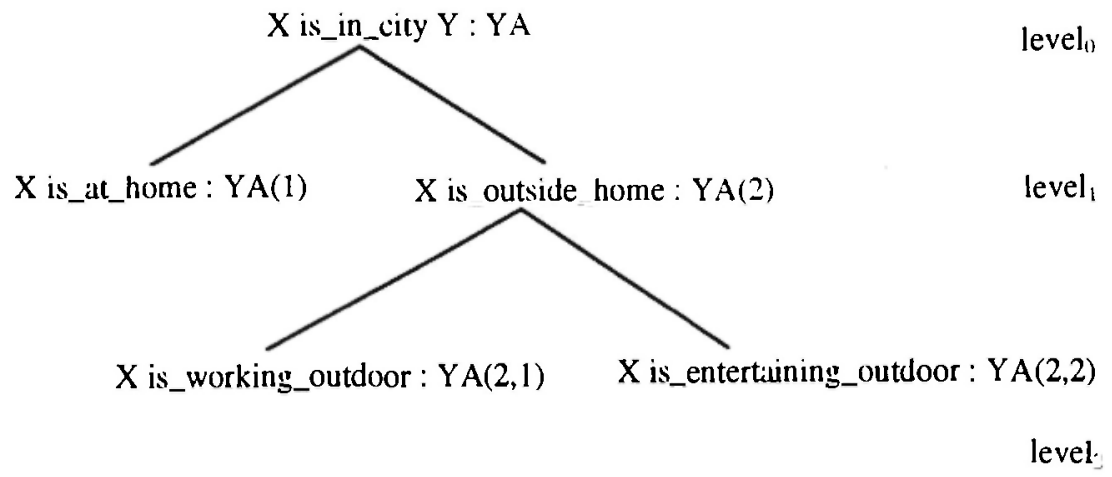

Fig. 1: An HCPRs-tree

\{level 0$\}$

Is_in_city $(X, Y)$ : YA

If [Lives_in_city $(X, Y): 1]: 0.85$

Unless [Is_on_tour $(X): 0.08$ ]

Generality [ ]

Specificity [Is_at_home $(X)$, Is_outside_home $(X)]$

$\{$ level 1$\}$

Is_at_home $(\mathrm{X})$ : $\mathrm{YA}(1)$

If [Time(night) :0.9] : 0.88

Unless [Is_doing_overtime $(X): 0.04$, Works_in_night_shift $(X): 0.03$ ]

Generality [Is_in_city $(X, Y)]$

Specificity[ ]

Is outside home $(\mathrm{X}): \mathrm{YA}(2)$

If [Time(day) : 1] $: 0.85$

Unless [Is_ill(X) : 0.04, Watching_TV(X): 0.015, Bad_weather : 0.03]

Generality [Is_in_city $(X, Y)$ ]

Specificity[Is_working_outdoor(X), Is_entertaining_outdoor(X)] 
\{level 2\}

Is_working_outdoor $(\mathrm{X}): \mathrm{YA}(2, \mathrm{I})$

If [Day(working) : 0.9] :0.88

Unless [National_holiday : 0.03 , Is_unemployed $(X): 0.03$ ]

Generality [ Is_outside_home(X)]

Specificity []

Is_entertaining_outdoor $(\mathrm{X}): \mathrm{YA}(2,2)$

If [Day(Sunday ) : 1] : 0.82

Unless [Met_an_accident $(X): 0.04]$

Generality[ Is_outside_home $(X)]$

Specificity[ ]

Here, any HCPR is a more specific case of its parent. Thus, the root HCPR represents the most general concept. Once this is verified, we can descend to its children for more specific information, depending upon our requirement and resources available. If any HCPR we reach is blocked, either because one of the preconditions is false or one of the censor conditions is true, any further search of its children is not necessary. Obviously, as the concept becomes more specific, the number of elements of its IF_set increases. It is not required, however, to list all such elements along with the HCPR because total inheritance is an inherent feature of the HCPRs-tree structure; each HCPR inheriting the entire $\mathrm{IF}_{-}$set of its parent and thus of all its ancestors. The computation of the certainty factor of the decision at the $i^{\text {th }}$ level of specificity under forward chaining of reasoning involves the following formula (in the following $D_{i}$ - decision at the $i^{\text {th }}$ level; $H C P R_{i}$ HCPR at the $i^{\text {th }}$ level, and so on):

$\mathrm{CF}\left(\mathrm{D}_{\mathrm{i}}\right)=\min \left(\mathrm{CF}\left(\mathrm{D}_{\mathrm{i}-1}\right), \mathrm{CF}\left(\right.\right.$ preconditions for $\left.\left.\mathrm{HCPR}_{\mathrm{i}}\right)\right) *$ Strength of implication where

Strength of implication $=\mathrm{CF}\left(\mathrm{HCPR}_{\mathrm{i}}\right)+$ Sum of the $\mathrm{CFs}$ associated with the CENSOR conditions in $\mathrm{HCPR}_{\mathrm{i}}$.

Note that depending on the resource constraints and the user requirements on certainty/specificity, all censor conditions are evaluated, or some are evaluated, or censors are totally ignored. For details see Bharadwaj and Jain (1992). 
Example : Consider the above set of HCPRs with the numerical estimates of CFs. and the corresponding HCPR-tree shown in Fig. 1. Suppose that the certainty factor of the premise 'Lives_in_city $(\mathrm{X}, \mathrm{Y})$ ' is 1 , and if censor condition 'is_on_tour' is

- false $\mathrm{YA}=1.0 *(0.85+0.08)=0.93$

- unknown $\mathrm{YA}=1.0 *(0.85+0.0)=0.85$

- true $\mathrm{YA}=1.0 * 0.0=0.0$. Also $\mathrm{YA}(1), \mathrm{YA}(2), \mathrm{YA}(2,1)$ and YA(2,2) will all become zero.

Further assuming $\mathrm{CF}$ ('Is_in_city $(\mathrm{X}, \mathrm{Y}))=\mathrm{YA}=0.93$, $\mathrm{CF}($ Is-outside_home $(X))=\min (0.93,1) *(0.85+(0.04+0.015+0.03))=0.86$.

Specificity and certainty are inversely related; we can gain specificity at the expense of certainty, or vice-versa. A General Control Scheme (GCS) has been suggested that may generate different control schemes that are based on the given values of some control parameters (see Bharadwaj \& Jain, 1992 ). GCS provides a reasoning mechanism that makes controllable trade-off between certainty and specificity. Fusion and Fission algorithms (Bharadwaj $\&$ Jain, in press) facilitate automatic restructuring to ensure consistency and minimum redundancy. Figures 2 and 3 illustrate the concepts of Fusion and Fission.

Many features that are related to requirements on a general representation scheme (Brachman \& Levesque, 1985; Michalski, \& Winston, 1986; Jain \& Bharadwaj, in press), namely, variable precision logic and general control scheme(GCS) (Bharadwaj \& Jain, 1992), small rule repair with Unless operator (Michalski, \& Winston, 1986; Bharadwaj \& Jain, 1992), genetic algorithms (Hewahi, 1994; Hewahi \& Bharadwaj, 1996), parallelization of reasoning (Varshneya \& Bharadwaj, 1994; Bharadwaj \& Varshneya, 1995), application of Demster-Shafer theory (Bharadwaj et al., 1994), fuzzy calculus (Neerja \& Bharadwaj, 1996), and so on, have already been addressed in the HCPRs system. An extended HCPRs system is discussed in the following section.

\section{EXTENDED HIERARCHICAL CENSORED PRODUCTION RULES (EHCPRS) SYSTEM}

The obvious problem for AI is how to characterize, to represent, and to compute with prototypes connected to psychology, or to concepts like natural 
Vol. 9, No. 3-4, 1999
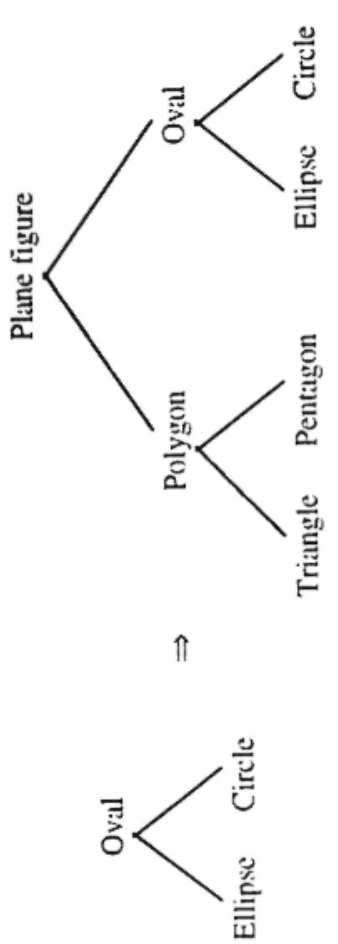

(త)

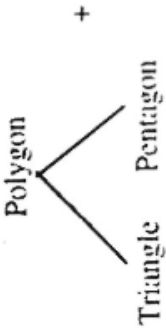

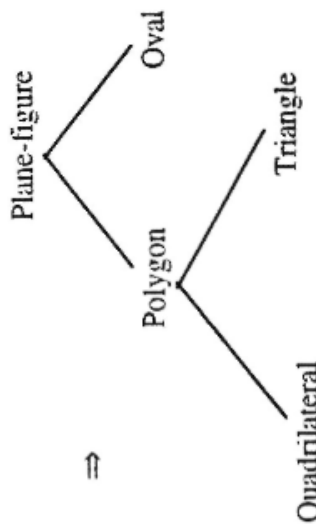

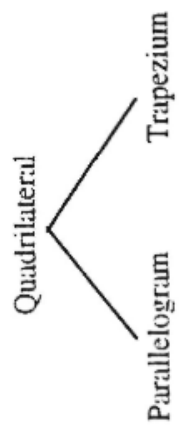

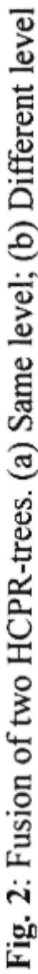

\section{$\widehat{a}$}

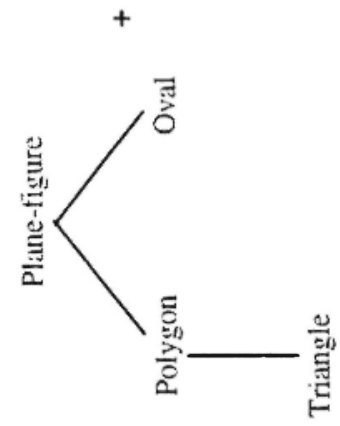


N.K. Jain, K.K. Bharadwaj,

N. Marranghello
Journal of Intelligent Systems

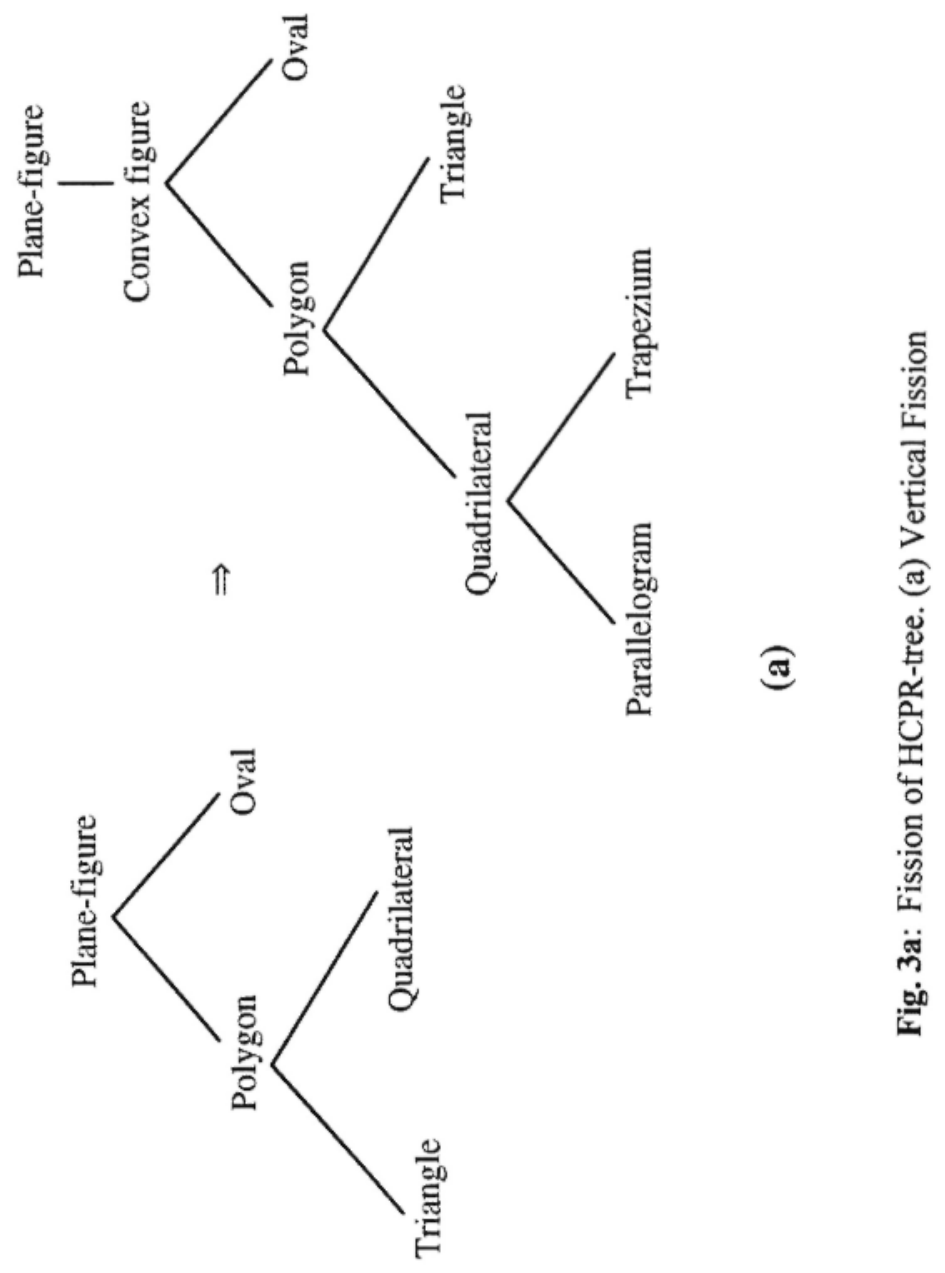




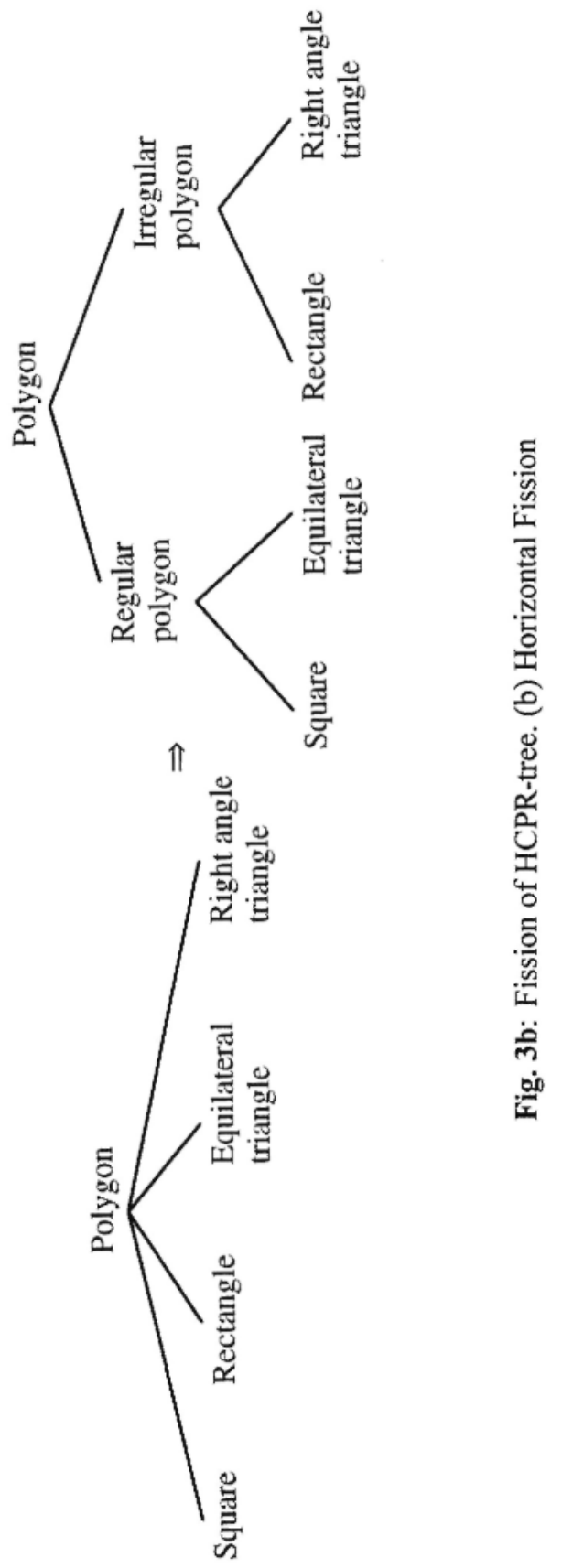


kinds in philosophy, where default assumptions play a prominent role (Reiter, 1987). A concept in general is assumed to possess two types of features, namely, defining features and characteristic features. Defining features of a concept must be true if an item or individual satisfies (or is a member of) the particular concept (or category/class) and must be relegated with the If operator using the appropriate relation (conjunction/disjunction) among the defining features. On the other hand, the characteristic features usually hold true for an item that is a member of a particular category, but is not necessary for the definition of that category. This class of features must be represented distinctly from the defining features represented with the If operator. In order to distinctly represent characteristic features, two new operators, namely, Has_part and Has_property are introduced. The features relegated with these two operators are allowed not to hold (and considered defaults) for an item or individual that is an instance or member of the particular category or class represented by the head of the EHCPR. It means that even if for an unknown object, one or more properties associated with these operators are not satisfied (or unknown), that particular EHCPR for the object would still be applicable. Hence the properties associated with these operators can be treated as cancelable. In this system, information about an item or an individual belonging to a concept may be represented using two different operators, namely the Has_instance and Instance_of operators. With Has_instance, all known individuals or items that are particular instances of that concept are relegated, whereas with the Instance_of operator, the concept of which of these individuals or items are members is represented. For example, if $\mathrm{C} 1, \mathrm{C} 2$ and $\mathrm{C} 3$ are particular instances of a concept $\mathrm{C}$ then

C Has_instance $(\mathrm{Cl}, \mathrm{C} 2, \mathrm{C} 3)$,

Cl Instance_of ( C),

C2 Instance_of $(C)$ and

C3 Instance_of( $C$ ) would hold.

With the inclusion of the new operators, the extended HCPRs (hereafter called EHCPRs) system can be defined as consisting of three major parts, namely, the static knowledge base or background knowledge structure, dynamic data base or set of instances (or data items), and an EHCPRs subsystem (similar to an expert system shell or kernel of a knowledge representation language) as an interpreter and manager (Fig. 4). The static knowledge base, similar to human long term memory, consists of knowledge which is assumed to become (after sometime of its creation) almost invariable 
with change in time and is consisting of all an EHCPRs representing general rules, definitions, or structures which are independent of particular domain instances.

\begin{tabular}{|c|c|c|c|}
\hline \multicolumn{2}{|c|}{$\begin{array}{l}\text { Knowledge Base } \\
\text { (Set of EHCPRs) }\end{array}$} & \multicolumn{2}{|c|}{$\begin{array}{c}\text { Data Base } \\
\text { (Set of data items) }\end{array}$} \\
\hline $\begin{array}{c}\text { User } \\
\text { Interface }\end{array}$ & $\begin{array}{l}\text { Ler } \\
\text { Proc }\end{array}$ & $\begin{array}{l}\text { ing } \\
\text { dures }\end{array}$ & $\begin{array}{l}\text { Reasoning } \\
\text { Procedures }\end{array}$ \\
\hline \multicolumn{4}{|c|}{ (EHCPRs - Subsystem) } \\
\hline
\end{tabular}

Fig. 4: Block Diagram of EHCPRs-System

An EHCPR takes the following form:

Concept $/ *$ as Head of rule */

If (Preconditions): (augmented with a numerical factor)

Unless (Exceptions):(augmented with some numerical factors)

Generality (General Concept)

Specificity (Specific Concepts)

Has_part (default: structural parts)

Has_property (default: characteristic properties)

Has_instance (instances)

(Notice that HCPR defined at [2.1] in Sec. 2 is included in the above extended definition of EHCPR and, therefore, all earlier results concerning the HCPRs system automatically hold true for an EHCPRs system. Hereafter, earlier references to HCPRs system and HCPR-tree will be referred to as EHCPRs system and EHCPR-tree).

The dynamic data base is similar to the short term memory of human beings, which is subject to major changes with change in time and location and contains information or data derived by the system through its interaction with the outside world in relation to its permanent background knowledge. The dynamic data base is similar to the facts of production system or the data base of predicate calculus based systems. Each data item in the data base will also have a uniform structure, having the following general form:

Head ( particular instance of a concept/name of individual object)

Instance_of (a general concept)

Has_part (set of actual known parts)

Has_property (set of known true properties) 
The data item defined above is reminiscent of Brachman and Schmolze's (1985) use of "Individual concept" in language KL-ONE for the individual description, in which each individual concept is said to individuate some generic concept. Here, the equivalent of 'Individuate' is Instance_of operator. Role descriptions in the KL-ONE individual concept are actual fillers of the Roles inherited from its parent concept, whereas the equivalents of the Role description are Has_part and Has_property operators in (3.2) above). The difference here is that these operators are filled with only the override properties. Others, inherited properties, are assumed to be implicitly associated with it, via the Instance_of and subsequently by Generality operators. The procedural knowledge is separately relegated in the system via EHCPR subsystem (Reiter, 1987; Brachman \& Levesque, 1984). Further, the system follows the functional approach of knowledge representation in which the user cannot interact directly with the knowledge structure but rather with the system-defined operations, which are internally converted and acted on to the system's knowledge structure (Brachman \& Levesque, 1985).

Consider the following EHCPR from knowledge base for the general class of Birds:

Bird

If (possess: feathers) .98

Unless (feathers: artificial): .01

Generality (Vertebrate)

Has_part (legs: 2, eyes: 2, beak: 1)

Specificity (Bulbul, Parrot, Sparrow, Pigeon)

Has_property (fly: yes, sight : good, egg-laying : yes, habitat: nest)

Has_instance(Titu, Mithu)

and a data item in the data base for particular instance of class Bird:

Titu

Instance_of (Bird)

Has_part (legs: 1)

Has_property (fly: no) 
Consider a sentence that 'Titu is a bird having one leg and cannot fly'. The information 'Titu is a bird' is explicitly represented in the EHCPR of Bird, using Has_instance operator in (3.3), and using Instance_of operator in data item for Titu in (3.4). The override or peculiar properties of Titu are kept with the Has_part and Has_property operators of the data item. The other properties are implicitly inherited by Titu from the concept of Bird, Vertebrate, Animal, and so on, to which it is connected by the Instance_of and then through the Generality operator. The information about subsumed classes of Bird (for example, Bulbul and Parrot) is shown to be represented with the Specificity operator. The information, for example, vertebrate is a subsumed class of the class Bird is relegated with the Generality operator. So, in this way meta knowledge (namely, knowledge about knowledge) is captured through various operators in an EHCPRs system.

The approach taken in an EHCPRs system is to represent different classes of properties with distinct and already defined operators (for example, in the above EHCPR for a Bird [3.3]), prototypical features of bird such as 'typically birds fly' and 'typically birds are biped' would be represented with the Has_property and Has_part operators, respectively. Information about some non-typical instances of bird, such as 'Titu having one leg and cannot fly' is given with the Has_instance operator. The necessary properties to define a concept such as, 'to be a bird it must possess feathers' is recorded with the If operator. As possession of feathers is not the sufficient condition for the definition of bird, all exceptions to this rule would be recorded with the Unless operator.

\subsection{Structural Representation}

The various symbols/properties/meanings to define a concept or individual are represented by using the same general form as given by (3.1) and (3.2) above. Any exception to the rule will be kept with the Unless operator. The domain knowledge is represented as a set of different chunks, and each chunk of knowledge is represented using the same general form of EHCPR given by (3.1), where each chunk is shown to be partitioned via different operators and connected to different EHCPRs through these operators. The hierarchical relationship between these chunks is made explicit through the Generality and Specificity operators. The knowledge in an 
EHCPRs system thus takes the network form through the connection of EHCPRs of different domains via the If, Unless, Has_part, and so on, operators. And this knowledge assumes a tree (hierarchical) structure with connections through the Generality and Specificity operators (see Fig. 1 for the HCPRs-tree; hereafter referred to as an EHCPRs -tree).

In the sections that follow, efforts are made to establish a relation between the knowledge represented in an EHCPRs -tree form with the structural representation languages, which are based on semantic networks and frames.

\subsection{Translation of Semantic Network to HCPRs Form}

One of the oldest and easiest to understand knowledge representation schemes is the semantic network (Quillian, 1968), which is composed of nodes and links. Semantic networks are basically graphic depictions of knowledge that show a hierarchical relation between objects. The knowledge given in a semantic network form must be translated in an equivalent coding system. In this section, our aim is to consider an EHCPRs system of representation from the perspective of such a coding system.

Consider the statement 'John gives Mary a book' and its semantic net representation by node 'el', shown in Fig. 5. The equivalent representation of the event 'el' in an EHCPRs system is encoded as set of the following EHCPRs and data items.
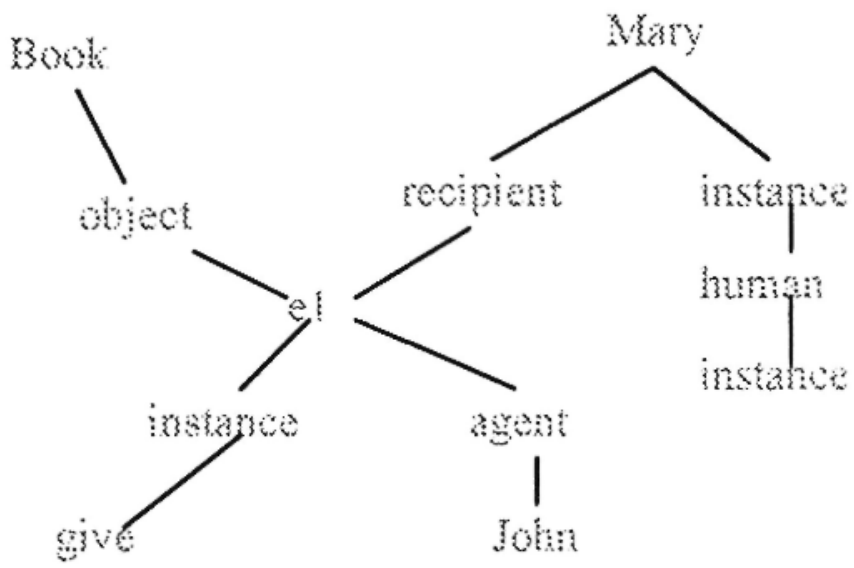

Fig. 5: Semantic Net representation of 'John gives Mary a book' 
/* Knowledge base*/

Human

Generality (Mammals)

If (Intelligence: highest)

Specificity (Child, Adult)

Child

Generality (Human)

If (Age: below 18)

Has_property (Age: positive integer, Address: string, Parents: (man, woman))

Specificity (Boy, Girl)

Boy

Generality (Child)

If (Sex: male)

Girl

Generality (Child)

If (Sex: female)

Book

Generality (Bound_Paper)

If (Has: number of printed bound pages)

Has_part (Body: no. of pages, Cover: (Hard or Soft)

Has_property (Title: string, Author/s: Name/s, Publisher : Address)

Give

Generality (Transfer)

If (receive (object, recipient) and performer (Agent))

Has_property (Object:, Recipient:, Agent:)

/* Data base */

el

Instance_of (Give)

Has_property (Object: Book, Recipient: Mary, Agent: John)

Mary

Instance_of (Girl)

Has_property (Age: 15, Address: 20 Down street, Parents: (Jack, Jill)) 
N.K. Jain, K.K. Bharadwaj,

N. Marranghello

John

Instance_of (Boy)

Has_property (Age: 16, Address: 320 New street, Parents: (Jim, Nick))

As another example consider semantic net representation of "Rover a dog guarded the John's house whole night carefully", as shown in Fig. 6.

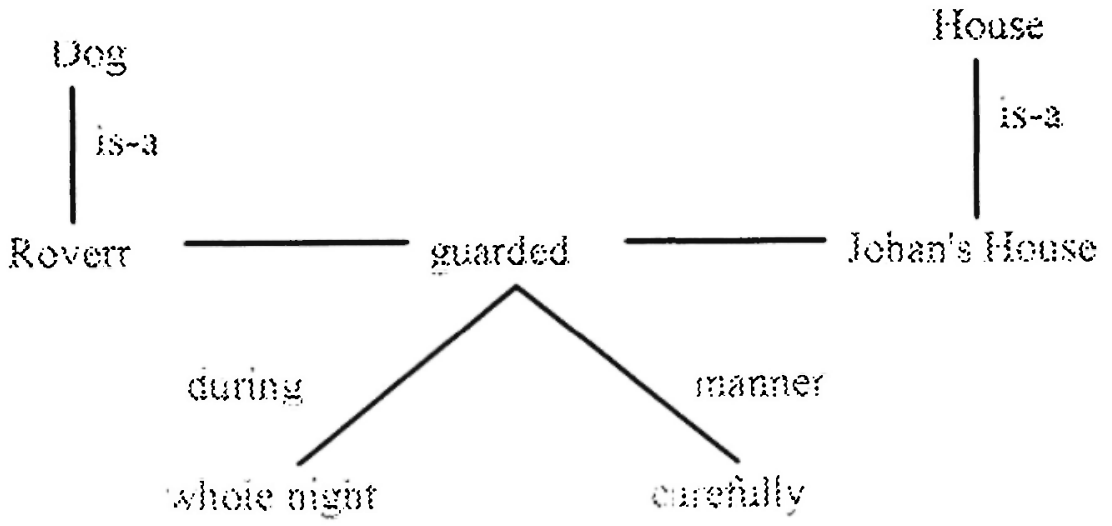

Fig. 6: Semantic net representation of "Rover a dog guarded the John's house whole night carefully"

The equivalent representation in an EHCPRs system are the following instances of EHCPRs. Here all the general concepts 'dog', 'watch', 'house', 'duration', and 'manner' are assumed to be present in the system as an EHCPRs:

/* Data base */

Rover

Instance of (dog)

Has_property (owner: John) 
John's house

Instance_of (house)

Has_property (owner: (John, Mary), Address: (27 Heaven street), Area: 2000 square feet)

whole night

instance_of (duration)

Has_property (Start time: start of night, End time: end of night)

carefully

Instance_of (manner)

Has_property (full of: care)

Guarded

Instance_of (Guarding)

Has_property (Agent: Rover, Object: John's house, duration: whole night, tense: past, manner : carefully)

\subsection{Translation of Frames to HCPRs Form}

Minsky (1975), in his influential frames paper, addressed the issues regarding prototypes, default assumptions, and definition of natural kinds, by suggesting the notion of a frame to represent stereotyped information. In a frame, the knowledge is organized in a special hierarchical structure that permits a diagnosis of knowledge independence and inheritance of properties.

Because of the complex syntax and the low modularity of frames, however, programming and using it for inference is difficult, and hence results in expensive software. Reasoning with frames is much more complicated than reasoning with production rules.

It is apparent that in frame system there is no equivalence of If, Unless and Specificity operators employed in an EHCPRs system. Moreover, in an instance frame, everything is to be copied from the general frames in the hierarchy. Whereas in equivalent data items in an EHCPRs system, only override properties and parts have to be recorded, and other general characteristics are implicitly associated through the Instance_of operator. It is shown below with an example of how can knowledge be represented using frame system be converted into a set of EHCPRs. Consider the following frame representation of "Earthquake". 
FRAME NAME EARTHQUAKE

SLOT1 PLACE: LOWER SLABOVIA

SLOT2 DAY: TODAY

SLOT3 FATALITIES: 25

SLOT4 DAMAGE: $500,000,000$

SLOT5 MAGNITUDE: 8.5

SLOT6 FAULT: SADIE HAWKINS

The suitable conversion of knowledge given by the above instance frame of earthquake in an EHCPRs system may take the following form:

/* Knowledge base */

Natural hazards

Generality (Bad events)

If (Agent: Nature)

Unless ()

Has_property (Fatalities: no of human died, Damage: value in dollars)

Specificity (Flood, Earthquake, Storms, Volcano_eruption)

Earthquake

Generality (Natural hazards)

If (Dislocation: earth crest)

Has_property (Magnitude: real number on rector scale Fault: address)

Has_instance (Earthquake_at_lower_Salbovia)

/* Data base */

Earthquake_at_lower_Salbovia

Instance_of (Earthquake)

Has_property (Place: Lower Salbovia, Time: today, Fatalities: 25, Damage: 500000000, Magnitude: 8.5, Fault: Sadie Hawkins) 
In the above translation of earthquake knowledge, only relevant data items and an EHCPRs are given, and other data items, say, place, time, damage, and so on, and an EHCPRs for Bad event, Flood, Storms, and so on, are assumed to be present.

\section{INHERITANCE AND RECOGNITION}

In this section, we will consider the notion of an EHCPRs-tree that is similar to the inheritance hierarchies presented by Shastri (1989). Inheritance is the form of reasoning that leads an agent to infer properties of a concept that is based upon the properties of its ancestors. For example, if the agent knows that birds fly, then given that "Sparrow is a bird" he may infer that "Chichi which is a Sparrow flies". This kind of reasoning, often referred to as default reasoning, is commonplace, and one can even argue that it is the quintessence of common-sense reasoning. In general, inheritance may be defined as the process of determining the properties of a concept ' $\mathrm{C}$ ' by looking up properties that are locally attached to ' $\mathrm{C}$ ', and if such local information is not available, then by looking up properties that are attached to concepts that lie above concept ' $\mathrm{C}$ ' in conceptual hierarchy.

In an EHCPRs system, each concept is represented by the head of a distinct EHCPR. The hierarchical relationship with other concepts is depicted using the Generality and Specificity operators. Relation between a concept and a data item is given by the Has_instance operator in an EHCPR and the Instance_of operator in a data item. A property is recorded with the highest EHCPR in an EHCPRs -tree to which it corresponds and is implicitly inherited by all descendant EHCPRs, unless the value of the override property is explicitly recorded with a EHCPR.

Once established or given, a concept may be employed to derive its relation with other concepts or attributes that are present in the system. In the process, new properties or characteristics of the concept may be identified. The process that incorporates this sort of reasoning in an EHCPRs system is referred to as the rules of inheritance with exceptions and is summarized below: 
Step 1: To determine a typical property or part not explicitly known about a given object, look at the EHCPR for that concept of which the object is given as an instance, namely, search Has_part for default parts and Has_property operator for default properties.

Step 2: If the information sought is not found at Step 1, then move up to the next parent EHCPR by making use of the information available with the Generality operator and apply the property of inheritance.

Step 3: Repeat Stepl and Step2 until the desired property is found or there are no more EHCPR up in the hierarchy, namely, the root of an EHCPRs -tree is reached.

Step 4: All the properties with If operators of an EHCPRs starting from present EHCPR of the concept to the root EHCPR via Generality operator would form necessary properties of the object.

Consider the following piece of knowledge:

Most Molluscs are shell bearer

All Cephalopods are Mollusc

Most Cephalopods are not shell bearer

All Nautili are Cephalopods

Nautili are shell bearer

All Naked Nautili are Nautili

All Naked Nautili are shell free (default)

(exception free)

(default)

(exception free)

(default)

(exception free)

(exception free)

In an EHCPRs system, this knowledge could be translated into the following form

Mollusc

If $(\mathrm{XI})$

Generality (Metazoan)

Has_part (back: shell)

Specificity (Cephalopod)

Nautili

If $(\mathrm{X} 3)$

Generality (Cephalopod)

Has_part (back: shell)

Specificity (Naked_Nautili)
Cephalopod

If $(\mathrm{X} 2)$

Generality (Mollusc)

Has_part (back: shell-free)

Specificity (Nautili)

Naked Nautili

If (back (shell-free))

Generality (Nautili)

Specificity () 
To be identified as an instance of Mollusc, an object must satisfy the property X1 given with the If operator of the EHCPR for Mollusc, as well as properties given with If operators of an EHCPRs that is higher in the EHCPRs -tree hierarchy, such as an EHCPRs for Metazoan, Animal, an so on. But at the same time, if it is known that the given instance has a back type as shell-free, the object can override the property given with the Has_part operator of the Mollusc. But if the back type is unknown, then by default the object will inherit the property from the Has_part operator of the Mollusc, namely, shell bearer.

Considering 'Moly' as a given instance of Mollusc., from the EHCPR of Mollusc the default statement 'Moly is shell bearer' and also the exceptionfree statement 'Moly must be an instance of Metazoan' may be inferred. Suppose that after some time, it is found that Moly is in fact an instance of Cephalopod, and its back type is not known explicitly, then we have to retrace our earlier default assumption and make a new default assumption that 'Moly is shell free'. This decision may be reversed any number of times, but all concepts that are specific to Naked_Nautili or to its instances must be shell free without exception.

The semantic networks are usually provided for defaults and their inheritance. But in their graphical notation, semantic networks do not distinguish at all between default rules and exception-free statements about the world. Such a distinction is captured in an EHCPRs system.

The recognition is the dual of the inheritance problem and, in an EHCPRs system, may be described as a process of finding a concept that matches best with a given description set, consisting of a list of properties that are supplied within the Has-part and Has_property operators.

Consider an unknown object 'Moly' described by the following sets of body parts and important properties.

Moly

Has_part (skin: yes, tail: no, Eyes: yes., ears: yes, Back: shell-free)

Has_property (eggs laying: yes, walk: yes, metazoan, X1, owner: John)

After applying the recognition algorithm to this unknown object, a data item may be appended to the data base having the following form : 
Moly

Instance_of (Mollusc)

Has_part (Back: shell-free)

Has_property (owner: John)

If the target for recognition step is set before hand as Mollusc, then it will result in successful recognition because the property XI (defining property with If operator in the EHCPR for Mollusc, above) is present in the description set, Has_property, for Moly. But if the target is set find whether Moly is cephalopod, then it will result in failure because of absence of the necessary property $\mathrm{X} 2$ (defining property with If operator in the EHCPR for Cephalopod, above) from the description set of Moly.

\section{HANDLING SEVERAL DEFAULT LOGIC PROBLEMS}

In default logic, as proposed by Reiter $(1978 ; 1980)$, the objective was to provide a representation for common sense facts of the form "typically A's are B's" and to articulate logic to characterize correct reasoning, using such facts.

Default theories, as described in Reiter (1987), are complicated (Reiter \& Criscuolo, 1980; Reiter \& Criscuolo, 1983). Whenever a new default rule is to be added to a representation, its potential interactions with other default rules is to be analyzed (Reiter \& Criscuolo, 1980). This analysis can lead to a re-representation of some of these defaults to block certain unwarranted derivations. Moreover, network representations, with respect to their ability to reason correctly with defaults, are shown to be deficient. The applicability of default logic, however, is limited by (a) the lack of intuitive semantics for the set of conclusions that the logic ratifies and (b) the high computational complexity involved in drawing such conclusions. Certain difficulties of default logic theory, as formalized by Reiter, are handled by translating default theories into classical prepositional theories (Ben-Eleyahu \& Dechter, 1996).

It is shown in this section how defaults are represented and various problems of interaction of defaults with other defaults or first-order formulae are handled in the proposed system. Semantic net and frames are suggested to represent and reason with natural kinds and usually provide for defaults and 
their inheritance. But semantic net and frames do not at all distinguish between default rules and exception free statements about the world. Consider an example of EHCPR for representation of class Elephant and a data item Clyde to represent member of class Elephant having three legs",

\section{Elephant}

Generality (Mammal)

If ( (Body part: Trunk) )

Has_part (legs:4)

Has_property (color: Gray)

Specificity (Royal_elephant)

Clyde

$$
\text { Instance_of (Elephant) }
$$

Has_part (legs: 3 ); This is a cancelable property

And a concept of "Triangle" with the non-cancelable property "three sides" would be represented in an EHCPRs system as:

Triangle

$$
\begin{array}{ll}
\text { If (sides: } 3) & \text {; it can not be cancelable } \\
\text { Generality (Polygon) } \quad \text {; all triangles are polygon } & \\
\text { Specificity (Equilateral, Isosceles, Scalene) }
\end{array}
$$

Consider the following example of two default rules, to discuss the problem with interaction of default rules :

Typically A's are B's or,

$$
\frac{A(x): M B(x)}{B(x)},
$$

Typically B's are C's or,

$$
\frac{B(x): M C(x)}{C(x)}
$$

Both are normal default rules. Default logic then admits the conclusion that "Typically A's are C' $s$ " in the sense that if ' $a$ ' is an individual for which $A(a)$ is known or believed, then $C(a)$ may be derived. In other words, normal default theories impose transitivity of typicality judgments. But these 
judgments may not be transitive, for example, from the following two rules:

"Typically high school dropouts are adults"

"Typically adults are employed"

We do not want to infer that "Typically high school dropouts are employed". So here transitivity needs to be blocked. In an EHCPRs system, this situation is handled by classifying features into two type of operators, namely., the operators for which transitivity is permitted and others that are non-transitive. In the proposed system, EHCPRs are assumed to be transitive with respect to If, Specificity and Generality part of the rule, whereas they are nontransitive with respect to Has_part and Has_property operators. Moreover, properties with an Has_part or an Has_property operator are subject to cancellation or overwrite. Yet, the definitional properties of the concept with If operator will remain unaltered and are never subject to cancellation. Hence the above statements might be converted into the following pair of EHCPRs.

High_school_dropouts

Generality (Dropouts)

If (Education: High School)

Has_property (Adult: yes)

Adult

Generality (Human)

If ( (Age: > 21))

Has_property (Employment Status: Employed, Marital Status: Married)

So from the above EHCPR for High_school_dropouts with the Has_property operator, at the most it may be inferred that 'Typically high school dropouts are adults', but the Has_property operator cannot inherit any property from the EHCPR for Adult, such as whether they are employed or married. Only properties which it can inherit are through its Generality operator and here it directs to EHCPR of Dropouts.

Next consider interactions between all and typically: This phenomenon is related to non-transitivity of interactions between normal defaults and certain universally quantified first order formula. Consider 
"All A's are B's" or, $\forall \mathrm{x} A(\mathrm{x})==>\mathrm{B}(\mathrm{x})$

and, “Typically B's are C's", namely

$$
\frac{B(x): M C(x)}{C(x)}
$$

From these two rules, default logic gives

$$
\frac{A(x): M C(x)}{C(x)}
$$

which is problematic. For example "All 21 years old are adults" and "Typically adults are married" would imply that typically 21 years old are married. But given that John is 21 years old, we would not want to conclude that he is married, as "all those who are 21 years old are married" is normally false. To overcome this situation, knowledge about adulthood can be represented in an EHCPR form as below:

\section{Adult}

\section{Generality (Human)}

If (Age : > 21 years)

Has_property (Martial Status: married, Employment Status: employed)

It is clear from the example above that in an EHCPR, the universal quantifier, All A's, is implemented using the If and Generality operators, whereas, quantification like most, many, a large number of, typically, (or existential quantifier), and so on, are implemented through the Has_part and Has_property operators. Form the above EHCPR for adult, it is easily inferable that if 'John' is an instance of adult, then 'John is married and employed' is assumed, unless stated otherwise. Nonetheless, it gives 'if John is 21 years old, he may be married', but not with $100 \%$ surety. Consider another example to represent and reason with the following knowledge:

"All Quebecois are Canadians"

"Typically Canadians are native English speaker"

"Typically Quebecois are native French speaker" 
N. Marranghello

The above rules may be translated into EHCPR form as below (EHCPRs at the beginning for Human and European are added for the sake of completeness):

Human

If (Live on : Earth)

Generality (Homo-sappiness)

Has_properties (Intelligence : Highest)

Specificity (European xor Asian xor American xor African)

European

If (Location : Europe)

Generality (Human)

Has_properties (Complexion : very fair to white)

Specificity (Canadian xor French xor German xor Spanish)

Canadian

Generality (European)

If (Nationality: Canada)

Has_property (Speak: English)

Specificity (Quebecois)

Quebecois

If (Domicile: Quebec)

Generality (Canadian)

Has_property (Speak: French)

In this example, 'all Quebecois are Canadian' is inferred using the Generality operator in the EHCPR for Quebecois, which has other implications as well. 'Typically Quebecois are native French speaker' is represented through the Has_property operator of the EHCPR of Quebecois. From the EHCPR of Quebecois and a given fact that 'John' is an instance of Quebecois, the following facts may be inferred unambiguously:

- John is having domicile of Quebec.

- John is having nationality of Canada.

- John is Canadian.

- John is European. 
Further the exclusive-or (xor) operator among different concepts within Specificity would allow us to infer:

- John is not Asian.

- John is not American.

- John is not Spanish

and so on.

In the above treatment of defaults in an EHCPRs system, the transitivity of properties is allowed via the Generality operator. Further, the analogous concepts are shown to be mutually exclusive in the Specificity operator (for example, a triangle and a quadrilateral are having an analogy that they are both polygons, but mutually exclusive, namely, an instance of a triangle can never be an instance of a quadrilateral). So at the particular instant of time, an individual cannot be allowed to be an instance of two or more conflicting concepts. Hence, it may be said that the problem of conflicting default assumption occurring in common instances is ruled out to a certain extent in an EHCPRs system.

\section{CONCLUSION}

In this paper, an Extended Hierarchical Censored Production Rules (EHCPRs) system has been proposed that employs a general representation having merits of logic, semantic networks, frames, and production rules. In this EHCPRs system, we have suggested a uniform or common code of representation in terms of an EHCPR. The uniform code of representation facilitates the development of general procedures for reasoning and learning (Bharadwaj and Jain, 1992; Hewahi, 1994; Jain, \& Bharadwaj, in press).

The various operators in an EHCPRs system support different forms of reasoning observed in real life. The results or information obtained from the If, Generality, and Specificity operators may be classified as hard-core knowledge, which would be reflected in real life from the phrases with $100 \%$ surety, dead sure, for all, and so on. Whereas the information obtained from the Has_part or the Has_property operators may be defeasible, which is differentiated in real life by using the phrases likely to have, by default, 
should have, might be having, there exist, most of, typically, and so on. A given chunk of knowledge is partitioned among different operators, and that gives rise to different interpretations and uses via distinct procedures in reasoning system for different operators.

We have discussed the problems of inheritance with the possibilities of both with cancellation and without cancellation, as well as recognition with partial match. Some default logic problems are shown to be handled in the proposed system. The notion of inheritance and recognition is generalized here as it also include reasoning about parts by making use of the Has_part operator separately, (Fahlman, 1979; Schubert et al., 1983). The notion of recognition and inheritance that is based on the principal of maximum entropy is formalized by Shastri (1989), and how numbers may be associated with symbolic representation and hence may be used in principled and effective manner is shown. Similarly in an EHCPRs system, a mechanism has to evolve for suitable augmentation of information that is relegated with different operators with numerical estimates of their likelihood and testing cost.

One of the most important and central points of distinction between an EHCPRs system and any other representation is in its ease of handling variable precision logic in general and exceptions in particular. Control planning is one of the important aspects that is incorporated into the system employing a General Control Scheme (GCS) (Bharadwaj \& Jain, 1992). The GCS describes how a reasoning mechanism depending on the control parameters (resource constraints and user requirements on certainty/ specificity) can make a controllable trade-off between a certainty of belief in a conclusion and its specificity. Another distinction may be on the efficient domain independent learning procedure in an EHCPRs subsystem.

Adaptability of the proposed system to "Genetic based machine learning" has already been explored (Bharadwaj et al., 1996; Hewahi, 1994; Hewahi \& Bharadwaj, 1996). Several efforts toward parallelization of reasoning in the system have been presented in (Bharadwaj \& Varshneya, 1995; Varshneya \& Bharadwaj, 1994). Application of fuzzy logic and Demster-Shafer theory has also been extended to the system (Bharadwaj et al., Goel,. 1994; Neerja \& Bharadwaj, 1996). One of the active future research directions would be the parallelization of possible learning algorithms and the application of multistrategy learning schemes in an EHCPRs system. It is to be seen that how this 
representational scheme incorporates temporal and spatial information into its knowledge structure naturally and efficiently.

\section{ACKNOWLEDGEMENTS}

This research is partially supported by the Brazilian foundation $\mathrm{CNPq}$ under Grant No. 301597/95-2. The authors feel very grateful to the anonymous referees of the Journal of Intelligent Systems for their valuable and encouraging comments.

\section{REFERENCES}

Ben-Eleyahu, R. and Dechter, R. 1996. Default reasoning using classical logic, Artificial Intelligence, 84, 113-150.

Bittencourt, G. 1990. An Architecture for Hybrid Knowledge Representation, Ph.D. Dissertation, University of Karlsrule, Department of Computer Science.

Bharadwaj, K.K. and Jain, N.K. 1992. Hierarchical Censored Production Rules (HSCPRs) system, Data \& Knowledge Engineering, 8, 19-34.

Bharadwaj, K.K., Hewahi, N.M. and Brandao, M.A. 1996. Adaptive Hierarchical Censored Production Rule-Based system: A genetic algorithm approach, in: Advances in Artificial Intelligence, SBIA'96, Lecture Notes in Artificial Intelligence, No. 1159, Berlin, Germany, Springer-Verlag, 81-90.

Bharadwaj, K.K., Neerja and Goel, G.C. 1994. Hierarchical Censored Production Rules system employing Dampster-Shafer uncertainty calculus, Information and Software Technology, 36, 155-164.

Bharadwaj, K.K. and Varshneya, R. 1995. Parallelization of hierarchical censored production rules, Information and Software Technology, 37, 453-460.

Brachman, R.J., Fikes, R.E. and Levesque, H.J. 1983. Krypton: a functional approach to knowledge representation, IEEE Computer, 16, 67-83.

Brachman, R.J., Gilbert, V.P. and Levesque, H.J. 1985. An Essential Hybrid Reasoning System: Knowledge and Symbol Level Accounts of Krypton, 
Proc. $9^{\text {th }}$ IJCAI, Los Altos, CA, USA, Morgan Kaufman.

Brachman, R.J. and Levesque, H.J. 1984. The tractability of subsumption in frame-based description languages, in: Proceedings of the American Association for Artificial Intelligence (AAAI).

Brachman, R.J. and Levesque, H.J. (editors). 1985. Reading in Knowledge Representation, Los Altos, CA, USA, Morgan Kaufmann.

Buchanan, B.G. and Shortliffe, E.H. 1984. Rule-Based Expert Systems, Reading, MA, Addison-Wesley.

Calmet, J., Tjandra, I.A. and Bittencourt, G. 1991. MANTRA: A shell for hybrid knowledge representation, In: Proceedings of the IEEE InterNational Conference on Tools for Al, San Jose, CA, USA.

Charniak, E. 1981. Common representation for problem-solving and languagecomprehension information, Artificial Intelligence, 16, 225-255.

Fahlman, S.E. 1979. NETL: A System for Representing and Using Real World Knowledge, Cambridge, MA, USA, MIT Press, 1979.

Guha, R.V. and Lenat, D.B. 1994. Enabling agents to work together, $C A C M$, $37,127-142$.

Haddawy, P. 1987 A variable precision logic inference system employing the Dempster-Shafer uncertainty calculus, MS Thesis (UILU-ENG-86-1777) University of Illinois, Urbana-Champaign, IL, USA, 1987.

Harmon, P. 1990. Object-oriented systems, Intelligent Software Strategies, September, 1990.

Hayes, P.J. 1977. On semantic nets, frames and associations, in: Proceedings of the $5^{\text {th }}$ IJCAI, Los Altos, CA, USA, Morgan Kaufmann, 99-107.

Hewahi, N.M. 1994. Genetic Algorithms Approach for Adaptive Hierarchical Censored Production Rules Based system, Ph.D. Dissertation, School of Computer and System Sciences, Jawaharlal Nehru University, India.

Hewahi, N.M. and Bharadwaj, K.K. 1996. Bucket brigade algorithm for HCPRs system, International Journal of Intelligent Systems, 11, 197226.

Jain, N.K. and Bharadwaj, K.K., Some learning techniques in Hierarchical Censored Production Rules (HCPRs) system, International Journal of Intelligent Systems, (in press).

Michalski, R.S. and Winston, P.H. 1986. Variable precision logic, Artificial Intelligence, 29, 121-146. 
Minsky, M. 1975. A framework of representing knowledge, in: The PsychoLogy of Computer Vision, edited by Winston, P.H., New York, McGrawHill.

Neches, R., Langley, P. and Klahr, D. 1987. Learning, development and production system, in: Production Systems Models of Learning and Development, edited by Klahr, D., Langley, D., and Neeches, R., MIT Press, Cambridge.

Neerja and Bharadwaj, K.K. 1996. Calculus of Fuzzy Hierarchical Censored Production Rules (FHCPRs), International Journal of Intelligent Systems, 2, 1-25.

Newell, A. and Simon, H.A. 1972. Human Problem Solving, Englewood Cliffs N.J., USA, Prentice Hall.

Quillian, M.R. 1968. Semantic memory, in: Semantic Information Processing, edited by M. Minsky, Cambridge, MA, USA, MIT press.

Reiter, R. 1978. On Reasoning by Default, in: Proceedings of TINLAP-2, Theoretical Issues of Natural Language Processing 2, University of Illinois, Urbana-Champaign, IL, USA, 210-218.

Reiter, R. 1980. A logic for default reasoning, Artificial Intelligence, 13, 81132.

Reiter, R. 1987. Nonmonotonic reasoning, Annual Review of Computer Science, 2, 147-186.

Reiter, R. and Criscuolo, G. 1980. On interacting defaults, in: Proceedings of the IJCAI-8I,Vancouver, BC, Canada, 270-276.

Reiter, R. and Criscuolo, G. 1983. Some representational issues in default reasoning, Journal of Computers \& Mathematics with Applications 9, 15-27.

Sawamoto, J. et. al., 1988. PROTON: a expert system tool on the PSI, in: Artificial intelligence development and applications, edited by Gero, J.S. and Stanton, R., Stanford Science Publishers, North Holland.

Schubert, L.K., Papalaskaris, and Taugher, J. 1983. Determining type, part, color and time relationships, IEEE Computer 16, 55-60.

Shastri, L. 1989. Default reasoning in semantic networks: a formalization of recognition and inheritance, Artificial Intelligence, 39, 283-355.

Smorynski, G. 1985. Self Reference and Modal Logic, Berlin, Germany, Springer Verlag. 
Thraisingham, B. 1989. Rules to frames and frames to rules, AI Expert, October, 1989.

Varshneya, R. and Bharadwaj, K.K. 1994. Hierarchical censored production rules: Some avenues for parallelization, First International Workshop on Parallel Processing, Dec 28-31, Indian Institute of Science, Banglore, India.

Waterman, D.A. 1970. Generalization learning techniques for the learning of heuristics, Artificial Intelligence, 1, 121-170.

Waterman, D.A. and Hayes-Roth, F.1978. An Overview of pattern-directed inference systems, in: Pattern Directed Inference Systems, edited by Waterman, D.A. and Hayes-Roth, F., New York, USA, Academic Press, 3-22. 